\title{
Editorial
}

\section{Clinical Neurophysiology: An Extension of Clinical Examination}

\author{
Rajib Nayan Chowdhury ${ }^{1}$, Mohammad Enayet Hussain ${ }^{2}$ \\ ${ }^{1}$ Associate Professor \& Head, Department of Neurophysiology, National Institute of Neurosciences \& Hospital, \\ Dhaka, Bangladesh; ${ }^{2}$ Associate Professor, Department of Neurology, National Institute of Neurosciences \\ \& Hospital, Dhaka, Bangladesh
}

\section{Background of Neurophysiology \\ "The old order changeth (changes), yielding place to new"-Lord Tennyson.}

The evolution in medicine has gone through changes. Most of the communicable diseases has come under control. New are emerging. Life expectancy has increased. Neurological disorders are becoming more common. Epilepsies and neuromuscular disorders constitute an important bulk. For diagnosis and management of these disorders clinical neurophysiology plays an important role. Clinical neurophysiology is a subspecialty of neurology that studies the central and peripheral nervous systems by recording electrical activity, either spontaneous or stimulated. It is actually an extension of a neurological examination. These tests measure the electrical functions of the brain, spinal cord, and nerves in the limbs and muscles. It can localize site, the type and degree of the lesion, along with revealing the abnormalities that are in query. In some countries it is a part of neurology or psychiatry, for example the United States and Germany. In other countries it is an autonomous specialty, such as Spain, Portugal, Italy, the United Kingdom, Finland, Sweden and Norway.

There are different types tests

- Electromyography (EMG) :Electrical activity is recorded by inserting needle in muscle.

- Nerve conduction studies (NCS): Electrical activity is recorded by stimulating peripheral nerves.

- Electroencephalogram (EEG): useful in evaluating seizures and various abnormalities of the cerebral cortex.

- Evoked potentials: This test evaluates specific tracts of the central and peripheral nervous system. These include visual, auditory, or somatosensory evoked potentials. These record the electrical responses of the brain and spinal cord to the stimulation of the senses.

- Polysomnography: A type of sleep study employed to diagnose disorders associated with abnormal sleep behavior

- Intraoperative monitoring, Intraoperative neurophysiologic monitoring surgery is done during DBS, Spinal, Aquastic neuroma.

History of NCS /EMG \& EEG: Luigi Aloisio Galvani (September 9, 1737 - December 4, 1798) was an Italian physician, physicist and philosopher discovered that the muscles of dead frogs legs twitched when struck by an electrical spark. This was one of the first forays into the study of bioelectricity. A field that still studies the electrical patterns and signals of the nervous system. The capability of detecting electromyographic signals improved steadily from the 1930s through the 1950s, researchers began to use improved electrodes more widely for the study of muscles. Clinical use of surface EMG (sEMG) for the treatment of more specific disorders began in the 1960s. Hardyck and his researchers were the first (1966) practitioners to use sEMG. In the early 1980s, Cram and Steger introduced a clinical method for scanning a variety of muscles using an EMG sensing device. So modern day machines has passed through stages of evolution. Whereas regarding EEG, in 1875, Richard Caton (1842-1926), a physician practicing in Liverpool, presented his findings about electrical phenomena of the exposed cerebral hemispheres of rabbits and monkeys in the British Medical Journal. The different scientist worked for development of techniques to record cortical activity. German physiologist and psychiatrist Hans Berger (1873-1941) recorded the first human EEG in 1924. The scope of using neurophysiological investigations had been started in Bangladesh in 1978 in the then IPGMR. Over the years the use has tremendously been increased \& now it is being used not only in the capital but also in the different hospitals of the country by different neurologist.

Utility of NCS/EMG: NCS/EMG plays pivotal role in diagnosis of neuromuscular disorders. Clinical EMG is composed of two main components- nerve conduction 
study \& needle EMG. These tests compliment each other. Additional electrodignostic procedures are $\mathrm{F}$ wave, $\mathrm{H}$ reflex, RNS and single fiber EMG. Neurologist doing EMG should have a good clinical background, must be efficient to run modern equipments, should know the normal values of nerves, motor unit action potential (MUAPS), should be aware of specific and non-specific electrodiagnostic findings in different neuromuscular disorders. Most important function is to localize the lesion-neuronopathy, radiculopathy, plexopthy, peripheral neuropathy, NM junction disorder or myopathy. It is also helpful to see the severity, progression, and prediction of prognosis.

Preparation for NCS \& EMG- some steps that are taken before doing the test-explain, history taking, examination, evaluating previous reports, setting up differentials then going for NCS/EMG. Thus establishing or refuting the neurological diagnosis. Synthesizing a new diagnosis is forbidden.

Utility of EEG: In EEG electrical activity of brain is recorded through electrodes placed over different parts of brain. Electrodes are of two types -surface \& depth. Electrodes are placed following the international 10/20 system. Main source of electrical activity is cortical cells, which are low voltage, under goes attenuation. Previous days EEG were of analog type, present day is digitalized. Arrangement of electrodes in different directions is called montages. The recorded electrical activities are artifacts, brain waves like Alpha, Beta, Theta \& Delta, normal \& abnormal EEG activities. Abnormal EEG activities are slow waves, attenuation and epileptiform activities. Epileptiform activities are spike / wave, sharp / wave. In generalized epilepsy epileptiform activities are found symmetrically over both hemispheres, in focal epilepsy at particular location e.g. temporal lobe. Spike are characterized by duration of 20-70 ms, whereas sharp is of $>70 \mathrm{~ms}$. EEG is important for classifying epilepsy, diagnostic in SSPE/JME/West syndrome, helpful in metabolic encephalopathy, CJD, before drug withdrawal and declaring brain death.

Others: RNS is important in pre \& post synaptic NM junction disorder. RNS is done over Median, Ulnar, and Accessory\& Facial nerve to see the decremental or incremental response. RNS is of two types Rapid $(30 \mathrm{~Hz}) \&$ Slow $(3 \mathrm{~Hz})$. As the patients cannot tolerate Rapid RNS exercise test is done. More than $10 \%$ decrement in postsynaptic or more than $100 \%$ increment in presynaptic is called significant response. Single fibre EMG (sEMG) is time consuming procedure done in RNS-negative ocular myasthenia.VEP, BAER, SSEP are evoked potentials tests done in labs to assess different tracts of CNS. Among these VEP is done to see any pre-chiasmallesion. In VEP P100 latency is measured, which is the first downward deflection of triphasic wave. P100 latency is prolonged in demyelinating lesion of optic nerve. It is helpful in differentiating between true blindness from malingering. So clinical neurophysiology is very important for the diagnosis \& management of patients with neuromuscular disorders and Epilepsy. These procedures has passed through advancement in developed world. Sleep studies and Intraoperative monitoring facilities are not available in all centres More neurologist, Neurosurgeons and technologist should be trained in our country to meet the challenges. [Journal of National Institute of Neurosciences Bangladesh, 2020;6(1): 1-2] 